\title{
Coupling of electronic and nuclear motion in a negative ion resonance: Experimental and theoretical study of benzene
}

\author{
Michael Allan, ${ }^{1, a)}$ (D) Roman Čurík, ${ }^{2, b)}$ and Petr Čársky ${ }^{2, c)}$ (D)

\begin{abstract}
AFFILIATIONS
'Department of Chemistry, University of Fribourg, Chemin du Musée 9, CH-1700 Fribourg, Switzerland

2]. Heyrovský Institute of Physical Chemistry, Academy of Sciences of the Czech Republic, v.v.i, Dolejškova 3, 18223 Prague 8, Czech Republic
\end{abstract} \\ a) Electronic mail: Michael.Allan@bluewin.ch \\ b) Electronic mail: Roman.Curik@jh-inst.cas.cz \\ c) Author to whom correspondence should be addressed: Petr.Carsky@jh-inst.cas.cz
}

\begin{abstract}
We present calculated and measured elastic and vibrational excitation cross sections in benzene with the objective to assess the reliability of the theoretical method and to shed more light on how the electronic motion of the incoming electron is coupled with the nuclear motion of the vibrations. The calculation employed the discrete momentum representation method which involves solving the two-channel LippmannSchwinger equation in the momentum space. The electron-molecule interaction was described by the exact static-exchange potential extended by a density-functional theory correlation-polarization interaction that models the molecular response in the field of the incoming electron. Cross sections were calculated for all 20 vibrational modes from near threshold until $20 \mathrm{eV}$. They were convoluted with a simulated instrumental profile for comparison with electron energy-loss spectra or appropriately summed for overlapping vibrations for comparison with measured cross sections plotted as a function of electron energy. An electron spectrometer with hemispherical analyzers was employed for the measurements. Good agreement of theory with experiment was obtained for the spectral profiles at $8 \mathrm{eV}$, and a nearly quantitative agreement was obtained at 3 and $4.8 \mathrm{eV}$. The theoretical results provided new insight into the excitation process, and it showed that more modes are excited than predicted by simple symmetry rules. Spectra showing the details of boomerang structure in the $1.15 \mathrm{eV} \pi^{*}$ resonance were recorded and are presented, although this aspect of experiment cannot be compared with the current theory.
\end{abstract}

Published under license by AIP Publishing. https://doi.org/10.1063/1.5110677

\section{INTRODUCTION}

A key issue in electron-driven chemistry is how the energy of the incoming electron is coupled to the motion of nuclei of a target molecule. Theoretical treatment of this process is greatly complicated by the transient nature of the intermediate negative ion (the resonance), given by the spontaneous loss of the electron. In this work, we evaluate the capacity of an improved version of the Discrete Momentum Representation (DMR) method ${ }^{1}$ to excite an elementary type of nuclear motion - the fundamental vibrations. We do this by comparing calculated and measured vibrational excitation cross sections.
The DMR method (i) provides also, apart from the energies and widths of the resonances, the cross sections for exciting all vibrational modes without a priori assumptions, (ii) is applicable even to large polyatomic molecules, and (iii) significantly improves the description of resonances by augmenting the exact static-exchange potential by a density-functional theory (DFT) correlation-polarization interaction. Our earlier work on cyclopropane ${ }^{1}$ demonstrated the power of the DMR method to quantitatively treat the problem and provided a deep insight into the excitation mechanism, without a priori assumptions about eliminating certain partial waves. It is thus more powerful than selection rules and correctly predicted and described even the excitation of 
unexpected modes, where the electron leaves in a p partial wave-that is, a wave other than the generally assumed possibilities of either the same wave as the incoming electron or an s-wave. ${ }^{2,3}$

We chose benzene for our study. It is a prototype of a molecule with a $\pi$ electron system, and it is also a prototype for vibrational excitation of polyatomic molecules by electron impact because it was on benzene that Wong and Schulz ${ }^{2}$ pointed out the great selectivity of the resonant excitation and rationalized this selectivity by selection rules, assuming that the scattered electron can leave the resonance either in the same partial wave in which it arrived or in an s-wave. Despite its prototype nature, there is not much theoretical work concerning vibrational excitation and only few quantitative experimental cross sections. Primarily, the elastic electron scattering was studied. For a comprehensive bibliography, we refer the reader to the most recent paper $^{4}$ on this topic.

Experimentally, several resonances were found in the energy range below $20 \mathrm{eV}$ by electron transmission spectroscopy (ETS). ${ }^{5}$ The lowest resonance was reported by different laboratories between 1.1 and $1.6 \mathrm{eV}$ (depending on whether adiabatic or vertical attachment energy was taken) and was interpreted as $\pi \mathrm{e}_{2 \mathrm{u}}$. Another resonance was observed by electron transmission spectroscopy at about $5 \mathrm{eV}$ and assigned ${ }^{5-7}$ as $\pi b_{2 g}$. It was also observed as a weak shoul$\operatorname{der}^{8,9}$ below $5 \mathrm{eV}$ on the plotted total cross section data. The $\pi$ resonances were later also observed as peaks in (relative) vibrational excitation cross sections (Fig. 40 of Ref. 10 and Ref. 11). Compilation $^{4}$ of electron scattering studies on benzene shows a broad peak observed in the region 7-13 eV.

The positions of the resonances and their assignments were reproduced by rigorous $a b$ initio calculations. ${ }^{4,12,13}$ Gianturco and Lucchese $^{12}$ computed the $\pi e_{2 u}$ resonance by the single-centerexpansion method at $1.82 \mathrm{eV}$, the $\pi \mathrm{b}_{2 \mathrm{~g}}$ resonance at 7.44 , and the $\mathrm{e}_{1 \mathrm{u}}$ resonance at $10.07 \mathrm{eV}$. Bettega et al. ${ }^{13}$ using the Schwinger multichannel approach reported the $\pi \mathrm{e}_{2 \mathrm{u}}$ resonance at $2.3 \mathrm{eV}$ and the $\pi \mathrm{b}_{2 \mathrm{~g}}$ resonance at $8.3 \mathrm{eV}$. Prajapati et al. ${ }^{4}$ used the R-matrix calculations and reported the $\pi \mathrm{e}_{2 \mathrm{u}}$ resonance at $2.66 \mathrm{eV}$ and the $\pi \mathrm{b}_{2 \mathrm{~g}}$ resonance at $7.41 \mathrm{eV}$, obtained from the energy dependence of the total cross sections. The effect of inelastic channels was simulated by an "absorption" potential.

In contrast to elastic scattering, experimental data on vibrational excitation of benzene by electron impact are scarce. After the pioneering papers by Schulz and collaborators, ${ }^{2,14}$ the only vibrational electron energy loss spectra (EELS) of benzene we found in the literature were those reported in the reviews by Allan ${ }^{10}$ for residual electron energies of $0.49,0.75$, and $1.10 \mathrm{eV}$ and by Kato and collaborators ${ }^{15}$ for the incident electron energy of $7.5 \mathrm{eV}$. We did not find in the literature any true $a b$ initio calculation of vibrationally inelastic scattering by benzene. Still, benzene was the molecule on which symmetry selection rules for vibrational excitation by resonant electron impact were formulated. ${ }^{2,3}$ The rules were based on symmetry considerations, namely, on the symmetries of fundamental modes and of the electronic states of the negative ion. The original selection rules by Wong and Schultz ${ }^{2}$ were based on symmetry considerations only. Gallup realized ${ }^{3}$ that the vibration excited by various resonances should be determined by inspection of the symmetry and also of the magnitude of S-matrix elements given in terms of integrals over vibrational functions and electronic states.
Several computational methods have been employed in calculations of the electron-impact vibrational excitation of polyatomic molecules. The complex Kohn variation technique was used by Rescigno et al. ${ }^{16}$ in their $\mathrm{CO}_{2}$ study. Dissociative electron attachment and the vibrational excitation of $\mathrm{CH}_{3} \mathrm{I}$ were explored with the singlepole R-matrix method. ${ }^{17}$ A number of calculations employed a single-center expansion technique for small polyatomic systems with different levels of approximations for the nuclear dynamics. The molecular systems considered by these computational studies were $\mathrm{H}_{2} \mathrm{O},{ }^{18} \mathrm{H}_{2} \mathrm{~S}^{19} \mathrm{CH}_{4},{ }^{20}$ and cyclopropane $\mathrm{C}_{3} \mathrm{H}_{6} .{ }^{21}$

The aim of the present work is to calculate the vibrational cross section for all modes quantitatively, in $a b$ initio manner, and to compare them to absolute experimental cross sections. The measurements automatically yielded, as a sideline, details of the boomerang structure in the $1.15 \mathrm{eV} \pi \mathrm{e}_{2 \mathrm{u}}$ resonance which we also present and discuss even though it is not addressed by the DMR method.

\section{EXPERIMENT}

The measurements were performed with a spectrometer using hemispherical electrostatic energy analyzers which has been described previously $y^{22,23}$ and does not require a detailed description here. The technical quality of the data is substantially improved in comparison with the older benzene measurements performed with a magnetically collimated spectrometer. ${ }^{10}$ The experimental conditions were the same as in our previous studies on cyclopropane ${ }^{1}$ and diacetylene. ${ }^{24}$ As in our previous studies, absolute values of the cross sections were determined by the relative flow technique and normalized to a theoretical helium elastic cross section. ${ }^{25}$ The confidence limit for the magnitudes of the inelastic cross sections is about $\pm 25 \%$ (two standard deviations). As in our previous work, we shall compare experimental and calculated data in two formats: (i) cross sections for a given electron energy loss, plotted as a function of the incident electron energy, which we shall call energy dependence spectra (EDS), and (ii) cross section plotted as a function of electron energy loss $\Delta E$ for a given incident electron energy.

\section{THEORETICAL MODEL AND COMPUTATIONAL DETAILS}

The computational method, which we use and which we call DMR (Discrete Momentum Representation), is a twochannel approach in the discrete momentum representation expressed for each vibrational mode by the following two matrix equations:

$$
\begin{aligned}
& T_{10}=U_{10}+U_{10} G_{0} T_{00}+U_{11} G_{1} T_{10}, \\
& T_{00}=U_{00}+U_{00} G_{0} T_{00}+U_{01} G_{1} T_{10},
\end{aligned}
$$

where 10 and 00 mean the transitions $1 \leftarrow 0$ and $0 \leftarrow 0$, respectively. Equations (1) and (2) represent a traditional expression for a two-channel scattering process, coupling in our case the elastic channel $\left(T_{00}\right)$ with the vibrational channel for a particular vibrational mode $\left(T_{10}\right)$. All integrals for the interaction potential operator $U$ are calculated rigorously in the $a b$ initio manner. For evaluation of $U_{10}$ and $U_{01}$ matrix elements, the harmonic vibrational functions were assumed. As in the theory of infrared (IR) spectroscopy, the integration over the vibrational coordinate was approximated by 
means of analytical derivatives of $U$ matrix elements with respect to normal coordinates at the equilibrium geometry. Details of the method and the numerically controllable approximations used have been described in our earlier papers. ${ }^{26,27}$ The code used in this work has been technically improved to provide higher numerical stability at low energies. For clarity, we note also here the approximation referring to coupling of the elastic and vibrationally inelastic channels in Eqs. (1) and (2) because it is relevant for the discussion in Secs. IV and V. It stems from our observation that cross sections for elastic scattering evaluated by means of Eq. (2) for different vibrational modes differ very little, and this fact permits us to simplify this equation as

$$
T_{00}=U_{00}+U_{00} G_{0} T_{00}
$$

and to obtain the $T_{00}$ matrix by a standard single-channel, often called fixed-nuclei calculation for elastic scattering. The $T_{00}$ matrix obtained by means of Eq. (3) is then used in Eq. (1) for all vibrational modes. Hence, the two-channel approach is so converted to a pseudosingle-channel approach where the size of all matrices is only $N \times N$ instead of $2 N \times 2 N$, where $N$ represents the number of planewave functions in the electronic basis. Next, by defining matrices $A_{11}$ and $B_{10}$ as

$$
\begin{aligned}
& A_{11}=\left(1-U_{11} G_{1}\right)^{-1}, \\
& B_{10}=U_{10}\left(1+G_{0} T_{00}\right)
\end{aligned}
$$

for each vibrational mode, we obtain the matrix of amplitudes as

$$
T_{10}=A_{11} B_{10} .
$$

The interaction matrices $U_{00}, U_{10}$, and $U_{11}$ are of the SEP (staticexchange plus polarization) type. The SE part is calculated rigorously in an $a b$ initio manner by using the Hartree-Fock density matrix. Its analytical derivatives with respect to coordinates of the target and dipole moment derivatives are obtained from coupled perturbed Hartree-Fock calculations. The short-range correlation potential is approximated by a model based on the DFT (density functional theory) as described previously. ${ }^{28}$ The long-range polarization part is determined by the static polarizabilities and their derivatives with respect to the nuclear coordinates. The static polarizabilities used in the present calculations were obtained as linear response functions in DFT calculations with PBE0 functional and Sadlej's polarized VTZ basis set ${ }^{29}$ as implemented in the program Dalton, Release 2.0 $(2005))^{30}$
A direct output of scattering calculations is a manifold of calculated vibrational cross sections which can be taken as a theoretical line electron energy loss spectrum. The obtained lines were positioned at values of experimentally determined frequencies. ${ }^{31} \mathrm{We}$ follow the conventional numbering and labeling of the 20 normal modes (10 of which, $v_{11}-v_{20}$, are doubly degenerate) of benzene. ${ }^{31}$ If not noted otherwise, for direct comparison with the experiment, we assumed a Gaussian shape for each line with a half-width of $15 \mathrm{meV}$ corresponding to the resolution of experimental measurements done in this paper. The heights of the peaks are set at the values of calculated differential cross sections. For degenerate modes, the cross sections were calculated for a single component of the normal mode only, and the result was multiplied by a factor of two.

It is important to note that the use of the procedure depicted by Eqs. (1)-(6) does not lead to the known adiabatic approximation $^{32}$ for vibrations as the present theory starts from the coupled two-channel system of Eqs. (1) and (2). Equations (1) and (2) represent a set of two coupled equations in the vibrational degrees of freedom (initial and final states), and thus, it cannot be considered neither the fixed-nuclei nor the adiabatic approximations. Although the later simplification of the theory in Eqs. (3)-(6) leads to the elastic events decoupled from the inelastic collisions (3) and thus resulting in a fixed-nuclei elastic theory, the inelastic T-matrix elements are not obtained by a simple average ${ }^{32}$ over the nuclear states involved.

A limitation of the DMR method in its present form is the use of the harmonic approximation. The consequence of this is that our theoretical model does not account for the excitation of overtones and combination bands. Nor does it account for phenomena caused by the coupling of electron and nuclear movements in the intermediate resonant state, which cause the boomerang structure in the low-energy $\pi \mathrm{e}_{2 u}$ resonance of benzene. We chose it because it is currently the only manageable way for treatments of polyatomic molecules. We obtained good results with it for cyclopropane ${ }^{1}$ and diacetylene. $^{24}$

\section{ELASTIC SCATTERING}

We begin our discussion with elastic cross sections because good results for elastic scattering are a prerequisite for successful evaluation of cross sections for vibrationally inelastic scattering in
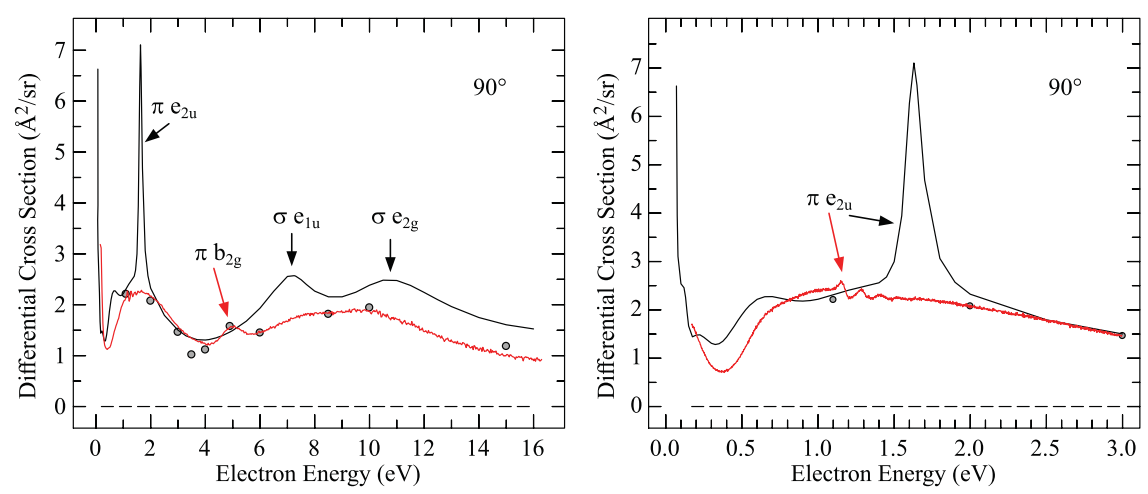

FIG. 1. Differential elastic cross section measured (red line) and calculated (black line) at a scattering angle of $90^{\circ}$ The experimental data were normalized to the absolute data of Cho et al. ${ }^{11}$ (shown as gray circles) at $6 \mathrm{eV}$. The experimental and the theoretical data are independently on absolute scales. The right panel shows the low energy region in detail. 
TABLE I. Differential elastic cross sections measured at $135^{\circ}$ by comparison with helium using the relative flow method.

\begin{tabular}{lccccccc}
\hline \hline Energy $(\mathrm{eV})$ & 0.2 & 0.4 & 0.8 & 1.0 & 1.15 & 2.5 & 5.0 \\
\hline $\mathrm{DCS}\left(\AA^{2} / \mathrm{sr}\right)$ & 0.975 & 1.330 & 0.986 & 0.680 & 0.999 & 1.014 & 1.618 \\
\hline
\end{tabular}

our approach. It can be seen from Eqs. (1) and (2) of our twochannel model that the $T_{00}$ amplitudes calculated for elastic scattering are needed for evaluation of the amplitudes of vibrational channels. As expected, our single-channel calculation (shown in the left panel of Fig. 1) almost duplicates the results of the calculations reported by Gianturco and Lucchese ${ }^{12}$ and Bettega et al. ${ }^{13}$ We have not measured the absolute values at $90^{\circ}$ and have normalized our elastic excitation function at this angle to the absolute value of Cho et al. ${ }^{11}$ at $6 \mathrm{eV}$. Figure 1 shows that with this normalization the present excitation function agrees with the values of Cho et al. ${ }^{11}$ also at other energies, confirming the consistency of the shape of the excitation function. At $135^{\circ}$, we have measured the absolute elastic values at several electron energies using the relative flow method, with the results listed in Table I, and show them in Fig. 2 as red, yellowfilled circles. The excitation function, corrected for the instrumental response function as in our earlier work, has been normalized to our absolute value at $1 \mathrm{eV}$. The good agreement between the yellow circles and the red line in Fig. 2 even at energies other than $1 \mathrm{eV}$ confirms the internal consistency of the absolute measurements and the excitation function. There is good agreement between the present absolute values and those of Cho et al., ${ }^{11}$ except of some discrepancy at 8.5 and $10 \mathrm{eV}$ at $135^{\circ}$.

Figures 1 and 2 compare the experimental cross sections to our calculation. The calculated position of the $\mathrm{e}_{2 \mathrm{u}}$ resonance, at $1.6 \mathrm{eV}$, agrees very well with the center of the boomerang structure of the experimental band (that is, with the vertical attachment energy) which is around $1.4 \mathrm{eV}$. This indicates correct treatment of polarization and short-range electron-molecule interactions by our theory.

The experimental curves, in particular the expanded views in the right panels of Figs. 1 and 2, show a clear-cut vibrational progression in the $\pi \mathrm{e}_{2 \mathrm{u}}$ resonance due to the boomerang mechanism. ${ }^{33}$ Our theoretical model describes the negative ion with fixed nuclei for elastic scattering and is consequently not amenable to treatment of vibrational motion in the negative ion. A meaningful comparison can nonetheless be made, based on the analysis by Chandra and Temkin, ${ }^{34}$ which they performed on the prototype case of vibrational excitation of $\mathrm{N}_{2}$ via the $\pi_{\mathrm{g}}$ resonance. They compare the results of the fixed nuclei theory, which does not reproduce the boomerang structure, with those of the "hybrid" vibrational-close-coupling theory, which does. They find that the ${ }^{2} \pi_{\mathrm{g}}$ resonance appears $0.4 \mathrm{eV}$ wide in the fixed nuclei approximation, which is about the "electronic" width $\Gamma$ of the resonance. This is also about the width of the individual boomerang peaks in the close-coupling theory. The whole envelope of the boomerang structure, which they calculated by the close-coupling theory, is about $1 \mathrm{eV}$ wide, in agreement with the experiment. The fixed nuclei peak is much higher than the boomerang structures such that the areas under the fixed nuclei peak and the boomerang peaks are comparable.

Given the findings of Chandra and Temkin, ${ }^{34}$ we conclude that our calculation reproduces not only the energy of the $\pi e_{2 u}$ resonance correctly but also approximately its magnitude if it is taken into account that the height of the narrow peak of the fixed nuclei level of theory is in the experiment distributed over several boomerang peaks.

The experimental cross sections in Figs. 1 and 2 show weak but clear structures around $5 \mathrm{eV}$ due to the $\pi \mathrm{b}_{2 \mathrm{~g}}$ resonance. Unexpectedly, this structure is missing in the theoretical elastic cross sections. This is consistent with earlier theoretical papers ${ }^{12,13}$ that do not show any peak or shoulder in the region from 3 to $8 \mathrm{eV}$. It will be shown below that despite the fact that the effect of this resonance on the elastic cross section appears to be underestimated by our theory, the theory is capable to describe its role for the vibrationally inelastic cross sections.

Our calculation further yields two distinct resonant peaks around 7 and $11-12 \mathrm{eV}$, in contrast to earlier theoretical papers ${ }^{12,13}$ where only one broad peak could be discerned in this energy range. These two resonances are of $\sigma \mathrm{e}_{1 \mathrm{u}}$ and $\sigma \mathrm{e}_{2 \mathrm{~g}}$ type, respectively. Their determination and their impact on the vibrational excitation of various modes will be discussed below. The experiment yields only one broad peak in the $7-12 \mathrm{eV}$ range at $90^{\circ}$, with a slightly flattened top indicating two unresolved peaks. The reasons for the absence of two distinct peaks could be that (i) the peak calculated at $11 \mathrm{eV}$ is at $10 \mathrm{eV}$ in the experiment and thus cannot be resolved from the $7 \mathrm{eV}$
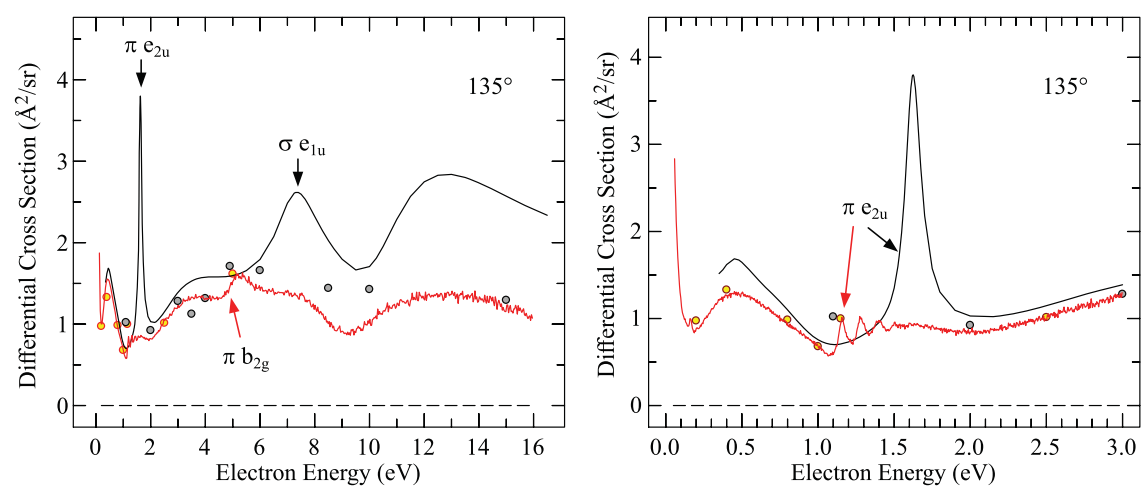

FIG. 2. Differential cross section measured (red line) and calculated (black line) at the scattering angle of $135^{\circ}$. Black, gray filled circles show the cross sections of Cho et al. ${ }^{11}$ (at $130^{\circ}$ ). Red, yellow filled circles are the present absolute values at $135^{\circ}$. All data are independently on absolute scale. The right panel shows the low energy region in detail. 
peak or (ii) that the potential curves of the negative ion are strongly repulsive, making the experimental peaks broader, by FranckCondon factors, than their electronic width alone. These peaks do not have a boomerang structure, the reasons presumably being that (i) their potential surfaces are likely to be repulsive and (ii) their electronic width is much larger than vibrational spacings. At $135^{\circ}$, two peaks are observed both in the experiment and in theory, around 7.5 and $13 \mathrm{eV}$. In terms of absolute values, good agreement between theory and experiment is found up to about $6 \mathrm{eV}$, but the calculated cross sections are larger than in experiment above this energy.

In particular, the $13 \mathrm{eV}$ feature is very broad and the question may be raised whether it is appropriate to denote it as a resonance. It is a question of semantics. It is known that every nonrelativistic Hamiltonian is fully described by a set of poles in a complex momentum (or energy) plane. These poles are the true resonances of the system ${ }^{35,36}$ [Eqs. (59) and (61) of Ref. 36]. The influence of the respective resonances on the cross section seen on the real energy axis depends on the distance of these poles from the real axis, represented by the resonance width. A Breit-Wigner fit to a distant pole's eigenphase will show a weak increase that is difficult to distinguish from the background formed by the other distant poles. There is no strict criterion to decide from what imaginary value of the pole we shall call it a "resonance" and below which it is just a "background."

\section{VIBRATIONAL EXCITATION: THEORY AND EXPERIMENT}

In this section, we compare theory and experiment for vibrational excitation. Since the current theory is limited to (i) the harmonic description of the vibrations of the target molecule and consequently cannot describe excitation of overtones and (ii) does not describe boomerang motion in the resonance, this section will be limited to excitation of fundamentals and disregard the boomerang structure. Overtone excitation and boomerang structure will be presented in Sec. VI.

There are two important ways to compare theory and experiment:

1. Comparison of cross sections for exciting selected specific vibrational modes, plotted as a function of electron energy (EDS). This method of comparison is particularly suitable for discerning the role of resonances, which generally cause enhancement of cross sections, that is, peaks in the spectra.

2. Comparison of energy-loss spectra recorded at given fixed incident electron energies. The spectra show all vibrational modes (albeit in some cases overlapping) and thus address an important strength of the present theory-the capacity to calculate cross sections for all modes. This way of comparison thus reveals the selectivity of excitation with respect to specific modes.

\section{A. Differential cross sections as a function of electron energy}

Theory has a decisive advantage over experiment-it provides cross sections for all modes separately, whereas vibrations with close-lying frequencies can often not be fully resolved in the experiment. This power of theory is illustrated in Fig. 3, presenting cross sections calculated for all 20 vibrational modes of benzene. It should be noted that all lines in Fig. 3 are open to uncertainties in the lowenergy range around the $\pi e_{2 u}$ resonance as the theoretical model used in this paper cannot account for the complicated nature of this resonance. This also applies to the theoretical EDS lines in Figs. 4 and 5. For the comparison with theory suitable superpositions of cross sections of overlapping vibrations need to be made as described in Secs. V A 1 and V A 2. The energy losses of 123 and $380 \mathrm{meV}$ were chosen for comparison. The former contains the ring stretch mode and is thus expected to be sensitive to $\pi^{*}$ resonances, where the temporarily occupied orbital has nodes between carbon atoms, making the potential surface repulsive with respect to the $\mathrm{C}-\mathrm{C}$ bond lengths. The $380 \mathrm{meV}$ energy loss is dominated by $\mathrm{C}-\mathrm{H}$ stretches and is expected to be sensitive to $\sigma^{*}$ resonances, with the temporarily occupied orbitals having nodes between carbon and hydrogen atoms and a potential repulsive with respect to the $\mathrm{C}-\mathrm{H}$ bonds. In this section, we also provide assignment of the symmetries to the resonances computed up to $11 \mathrm{eV}$ of collision energies. This assignment is carried out via analysis of the inelastic T-matrix elements as previously done by Čurík et al. ${ }^{1}$ More details will be given in a follow-up publication describing the role of the symmetries in electron-impact vibrational excitation of benzene.

\section{Energy loss of $123 \mathrm{meV}$}

Five vibrational modes contribute to the observed signal at the energy loss of $\Delta E=123 \mathrm{meV}: v_{2}, v_{6}, v_{7}, v_{14}$, and $v_{19}$, with the energies $123,125,123,129$, and $121 \mathrm{meV}$, respectively. Reference to Fig. 3 reveals that these modes include the totally symmetrical ring stretch $\left(v_{2}\right)$ and three $\mathrm{C}-\mathrm{H}$ bend modes. The left panel of Fig. 4 shows the calculated differential cross sections (DCS) for these five modes. It shows that the dominant contributions to resonant enhancement are due to the $v_{19}$ and $v_{7}$ modes. EDS plotted for the sum of all five calculated cross sections is compared with the experimental EDS in the right panel. (Simple sum, rather than the weighted sum, is taken because the frequencies of the five modes are close to each other.)

As in the plot for an elastic cross section, the peak for the first resonance in the calculated curve is narrower and higher than the experiment because of the neglect of vibrational motion in the transient anion. The right panel of Fig. 4 suggests a satisfactory agreement even for the $e_{2 u}$ resonance, but it should be taken with some caution. The theory suggests (left panel of Fig. 4) that the cross section is due primarily to excitation of $v_{19}\left(\mathrm{e}_{2 \mathrm{u}} \mathrm{C}-\mathrm{H}\right.$ bend), with negligible contribution of $v_{2}$ (symmetrical ring stretch), and, as already mentioned, these two modes cannot be resolved in the present experiment on $\mathrm{C}_{6} \mathrm{H}_{6}$. They were resolved in the $\mathrm{C}_{6} \mathrm{D}_{6}$ spectrum of Wong and Schulz, ${ }^{2}$ however, because the ring stretch vibration remains nearly unchanged at $117 \mathrm{meV}$, whereas the $\mathrm{C}-\mathrm{D}$ bend vibrations all drop to about $100 \mathrm{meV}$. The $\mathrm{C}_{6} \mathrm{D}_{6}$ spectrum indicates a prominent excitation of $v_{2}$.

A clear resonant enhancement is calculated at $4.2 \mathrm{eV}$, reasonably close to the experimental value of $4.8 \mathrm{eV}$. It is somewhat surprising that the resonance is calculated at a lower energy than measured because the experimental energy is lowered by 


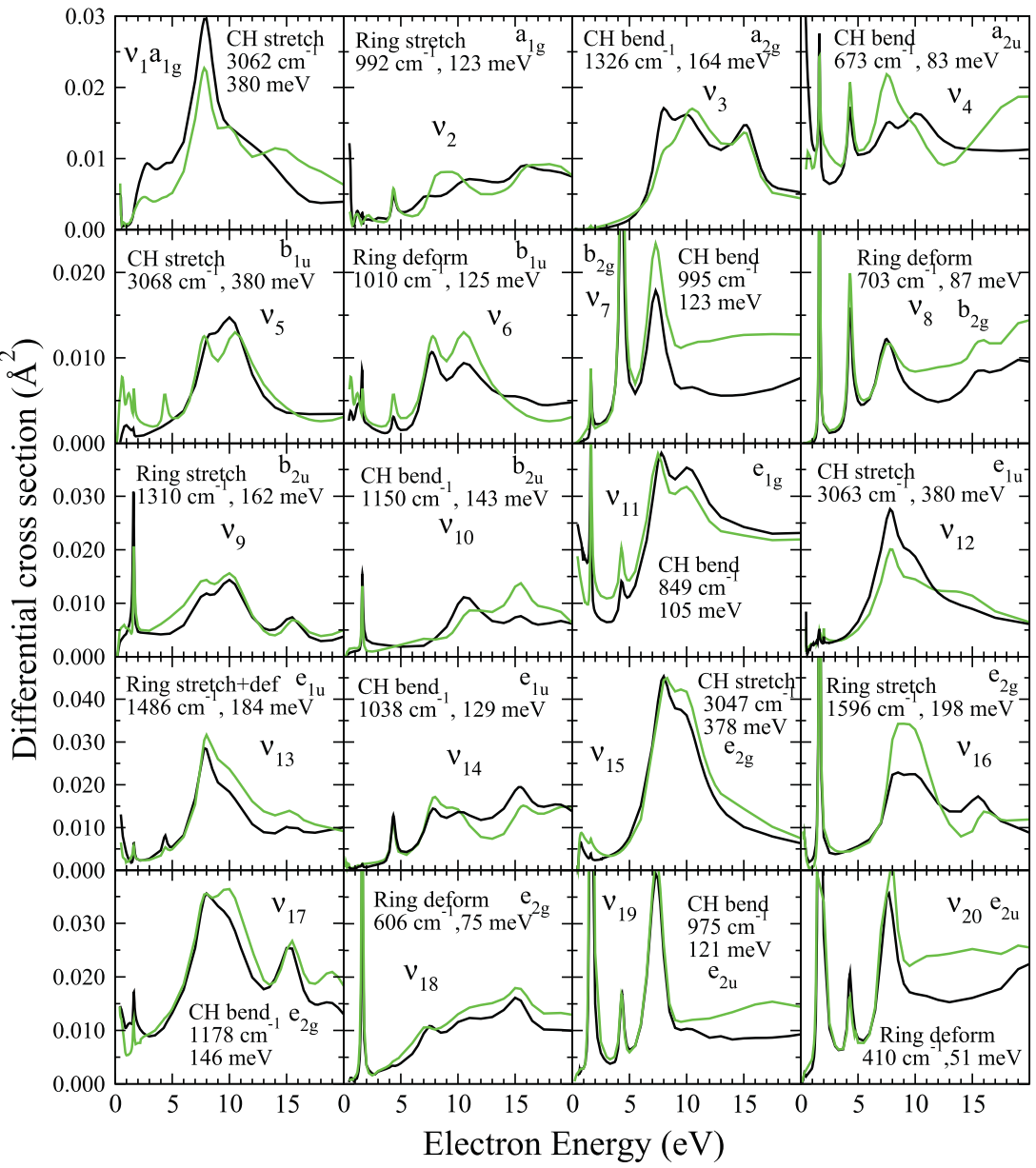

FIG. 3. Energy dependencies of the differential vibrational cross sections calculated for all 20 vibrational modes of benzene at the scattering angle of $90^{\circ}$ (black lines) and $135^{\circ}$ (green lines).

configuration mixing with an excited configuration, ${ }^{6,13}$ not included in the present calculation. The accuracy of the resonant positions is sensitive to the reliability of the DFT correlation-polarization interaction. It looks as though the DFT correlation tends to be somewhat too weak on the carbon centers, giving insufficient shift of the $e_{2 u}$ resonance, whereas it is probably too strong on the $\mathrm{C}-\mathrm{H}$ bonds and thus overshooting the $4.8 \mathrm{eV}$ resonance to lower energies.
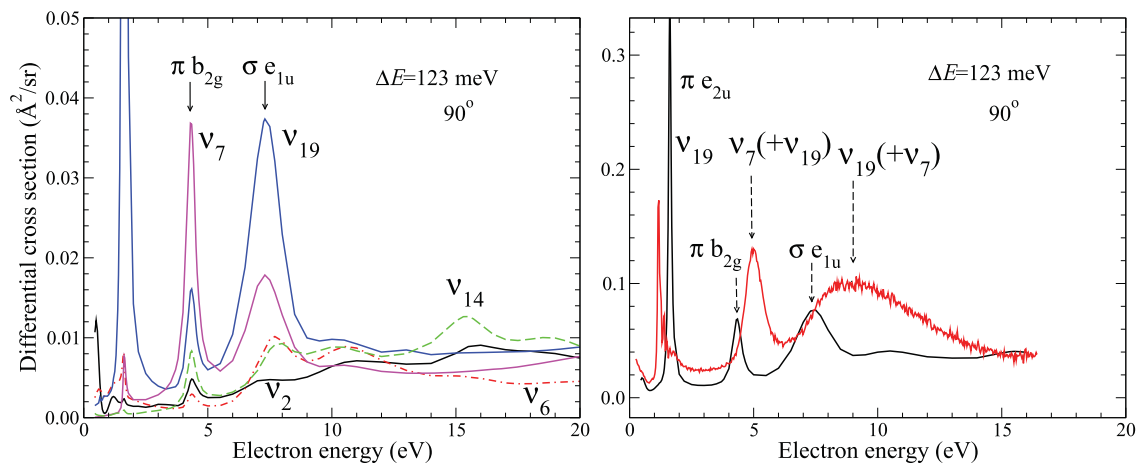

FIG. 4. Energy dependence of the differential cross section at the angle of $90^{\circ}$ and with the fixed energy loss of $123 \mathrm{meV}$. Left panel: calculated EDS curves for the five modes with frequencies in the $123 \mathrm{meV}$ range. Right panel: the black line represents the sum of all five contributions. The red line stands for the observed EDS. The assignment labels in the right panel indicate which modes make major contributions to the respective resonant enhancement. Mode $v_{6}$ is omitted because of its low cross section in the whole energy range. All curves are independently on absolute scales. 

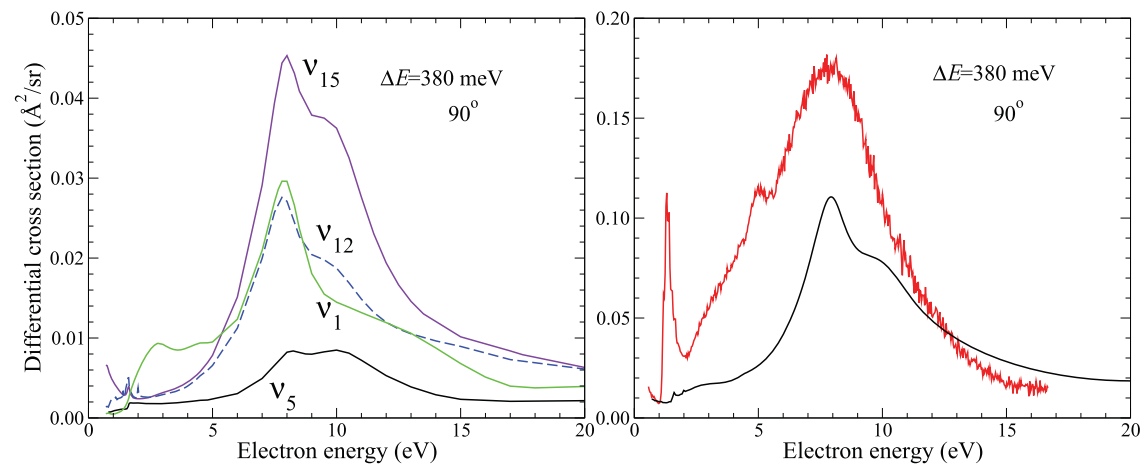

FIG. 5. Energy dependence of the differential cross section at the angle of $90^{\circ}$ and with the fixed energy loss of $380 \mathrm{meV}$. The left panel presents EDS curves for modes $v_{1}, v_{5}, v_{12}$, and $v_{15}$ with the frequencies $380,380,380$, and $378 \mathrm{meV}$, respectively. The black line in the right panel represents the sum of all four contributions. The red line stands for the observed EDS. All curves are on absolute scale.
The calculated $4.8 \mathrm{eV}$ peak is lower in absolute magnitude than that observed by the experiment, but taking into account that calculation of quantitative cross sections is generally a very difficult task because the cross section is a very steep function of the calculated parameters of the resonance, in particular, its width, the agreement may be classified as very satisfactory. The width of the $4.5 \mathrm{eV}$ peak also appears narrower in the theory $(0.5 \mathrm{eV})$ than in the experiment $(0.8 \mathrm{eV})$. This could be due to the fact that the calculated energy is lower than the measured and resonance widths generally steeply rise with rising energy. It is also likely that the potential curve is repulsive and the experimental width is a combination of the electronic width $\Gamma$ and the Franck-Condon width, which will intervene even in cases where there is no boomerang structure. The theoretical curve also reproduces qualitatively correctly the cross section above $6 \mathrm{eV}$ except that the two features calculated at 7.5 and $10.5 \mathrm{eV}$ are not resolved as individual peaks in the experiment but appear as one broad peak peaking at around $9 \mathrm{eV}$. This could be due to the larger width of the experimental peak which causes them to strongly overlap.

In conclusion, theory agrees well with experiment at energies above about $2 \mathrm{eV}$. At the $1.15 \mathrm{eV}$ resonance, theory reproduces the total vibrational excitation at $\Delta E=123 \mathrm{meV}$ satisfactorily but underestimates the excitation of the symmetrical ring stretch vibration $v_{2}$, which is revealed by the $\mathrm{C}_{6} \mathrm{D}_{6}$ spectrum of Wong and Schulz. ${ }^{2}$

\section{Energy loss of $380 \mathrm{meV}$}

The energy loss is fixed at $380 \mathrm{meV}$ in Fig. 5, and the observed signal is due to the four $\mathrm{CH}$ stretching modes $v_{1}, v_{5}, v_{12}$, and $v_{15}$, with the energies $380,380,380$, and $378 \mathrm{meV}$, respectively. Theory and experiment agree in that the cross sections for exciting the $\Delta E=380 \mathrm{meV}$ vibrations are dominated by very broad features in the $5-15 \mathrm{eV}$ energy range. Theory and experiment also agree reasonably well on the absolute magnitude of the cross section. Similar to the data in Fig. 4, two overlapping peaks appear around 8 and $10 \mathrm{eV}$ in the calculated cross section, but only one peak peaking around $8 \mathrm{eV}$ can be discerned in the experimental spectrum.

As can be seen in Fig. 3, theory predicts that the $\mathrm{e}_{2 \mathrm{u}}$ resonance at $1.15 \mathrm{eV}$ and the $\mathrm{b}_{2 \mathrm{~g}}$ resonance at $4.8 \mathrm{eV}$ are absent in the cross sections exciting these four vibrations at $90^{\circ}$ (the $4.8 \mathrm{eV}$ resonance is weakly present in the cross section for exciting $v_{5}$ at $135^{\circ}$ but not at $90^{\circ}$ ). The weaker (in terms of areas under the peaks) peaks appearing at these energies in the experimental spectrum in the right panel of Fig. 5 are thus likely to be due to the excitation of overtone vibrations, not accounted for in the theory. The behavior of the cross section for exciting $v_{1}$ in the $2-5 \mathrm{eV}$ range (left panel in Fig. 5) differs from the behavior of the cross section for exciting the other $\Delta E=380 \mathrm{meV}$ vibrations, in that it does not continuously drop to zero with decreasing energy below $5 \mathrm{eV}$ but retains a considerable value (around $0.01 \AA^{2} / \mathrm{sr}$ ) down to $2 \mathrm{eV}$. This behavior appears to be confirmed by the experiment-the cross section in Fig. 5 is sizable in the $2-4 \mathrm{eV}$ range. Explanation of this problem on theoretical grounds needs a detailed analysis of symmetries of resonance. The work along these lines is in progress.

\section{B. Electron energy-loss spectra}

\section{Incident electron energy of $3 \mathrm{eV}$}

At this energy, we obtained an almost quantitative agreement between theory and experiment, as can be seen in Fig. 6. In the case of band overlap, Fig. 6 lists only those modes which, according to the calculation, dominate a given observed peak. A weak peak at $-50 \mathrm{meV}$ is assigned to the $v_{20} 1 \rightarrow 0$ anti-Stokes transition, called a "superelastic peak" in the electron scattering community. Because of the reversibility principle, the height of this peak relative to the

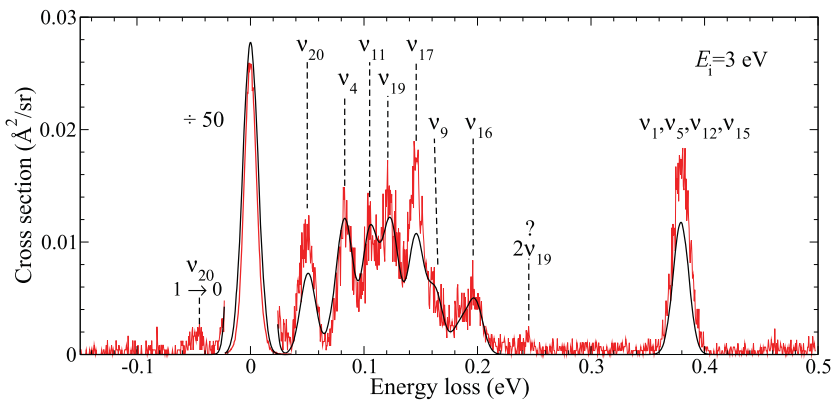

FIG. 6. Electron energy loss spectrum at the incident electron energy of $3 \mathrm{eV}$ and the scattering angle of $135^{\circ}$. The experimental spectrum is plotted in red, and the theoretical spectrum is plotted in black. Both spectra are plotted on absolute scale. Assignment of the observed peaks is assisted by the calculated cross sections (Fig. 3) in cases of ambiguity caused by peak overlap. 
inelastic peak is given by the Boltzmann factor for the abundance of the first excited vibrational state at room temperature. Numerically, it is $13 \%$, which corresponds to the ratio of heights of $1 \rightarrow 0$ and $1 \leftarrow 0$ peaks. As already mentioned above, five modes in the energy range of 121-129 meV form a peak at $123 \mathrm{meV}$. We designated it in Fig. 6 as $v_{19}$, as according to our calculations its contribution is the largest, although it is not dominant. The ratio of calculated cross sections is 1:2:3:2:5 in the series $v_{2}, v_{6}, v_{7}, v_{14}$, and $v_{19}$.

\section{Incident electron energy of $4.8 \mathrm{eV}$}

The spectrum for this electron energy, corresponding to the $b_{2 g}$ resonance, was published by Wong and $\mathrm{Schul}^{2}$ for $\mathrm{C}_{6} \mathrm{D}_{6}$. This resonance was characterized as short-lived, based on the broad peak without the boomerang structure in the electron transmission spectra of Nenner and Schulz ${ }^{6}$ and Burrow et al. ${ }^{7}$ Accordingly, it is amenable to treatment by our theoretical model. The agreement of theory with experiment is satisfactory shown in Fig. 7, although the calculated cross sections for most modes are underestimated. There is some overlap of peak due to vibration modes that are close in frequency. Hence, the theory suggests that the $v_{4}$ peak contains admixture of $v_{8}$ in the ratio 2:1. The strongest peak is predicted to be due to the $v_{7}$ mode, with the other four modes close in energy being 3-4 times weaker. Wong and $\mathrm{Schulz}^{2}$ also assigned the strongest peak in their $\mathrm{C}_{6} \mathrm{D}_{6}$ spectrum to the $v_{7}$ mode with $\mathrm{b}_{2 \mathrm{~g}}$ symmetry and assigned the peak at twice this frequency to the $2 v_{7}$ transition. Wong and Schulz ${ }^{2}$ designated the CD stretching peak as $v_{1}\left(\mathrm{a}_{1 \mathrm{~g}}\right)$. Our calculations for $\mathrm{C}_{6} \mathrm{H}_{6}$ predict that all four $\mathrm{CH}$ stretching modes provide appreciable contributions to the $380 \mathrm{meV}$ peak (in the ratios 5:3:5:6 for $v_{1}, v_{5}, v_{12}$, and $v_{15}$ ).

There are five vibrational modes close in energy in the $123 \mathrm{meV}$ region. The two strongest peaks, $v_{7}$ and $v_{19}$, are of about the same intensity. The peak at $241 \mathrm{meV}$ is assigned tentatively to $2 v_{7}$.

\section{Incident electron energy of $8 \mathrm{eV}$}

Figure 8 shows excellent agreement between the observed spectrum and the calculated profile of vibrational excitation. The calculated elastic cross section is slightly larger than the measured, as already pointed out in Sec. IV and shown in Fig. 2.

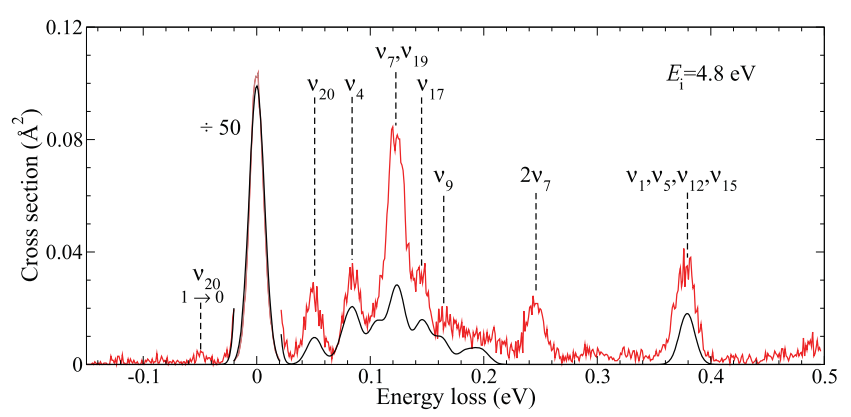

FIG. 7. Electron energy loss spectrum of $\mathrm{C}_{6} \mathrm{H}_{6}$ at the incident electron energy of $4.8 \mathrm{eV}$ and the scattering angle of $135^{\circ}$. The experimental spectrum is plotted in red, and the theoretical spectrum is plotted in black. Both spectra are plotted on absolute scale. In the case of overlapping modes, the labels indicate the mode with the largest calculated cross section.

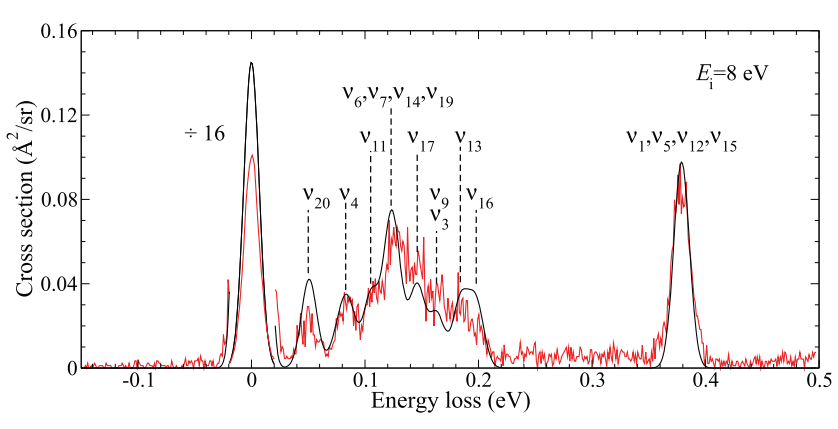

FIG. 8. Electron energy loss spectrum for the incident electron energy of $8 \mathrm{eV}$ and the scattering angle of $135^{\circ}$. The experimental spectrum is plotted in red, and the calculated one is plotted in black. Both spectra are plotted in absolute scale. In the case of overlapping modes, the labels indicate the mode with the largest calculated cross section.

As previously mentioned, in the case of overlapping modes, the labels in Fig. 8 indicate the mode(s) with the largest calculated cross sections. Theory suggests that the peak labeled $v_{4}$ also contains contribution from the $v_{8}$ mode (in the ratio 2:1) and that $v_{3}$ and $v_{9}$ are of about the same calculated intensities. The highest peak is due to five modes, $v_{2}, v_{6}, v_{7}, v_{14}$, and $v_{19}$, with the ratio of their predicted intensities $0.7: 1: 2: 2: 3$. The $v_{10}$ peak is overlapped by the 10 times stronger $v_{17}$ peak. All four $\mathrm{CH}$ stretching modes contribute to the peak at $380 \mathrm{meV}$. The calculated intensities for the $v_{1}, v_{5}, v_{12}$, and $v_{15}$ modes are in the ratio 2:1:2:4. In contrast to the spectra recorded for 3 and $4.8 \mathrm{eV}$, we do not see an overtone at $245 \mathrm{meV}$ and the "superelastic" peak at $-51 \mathrm{meV}$.

\section{BOOMERANG STRUCTURE OF THE $\pi^{*} e_{2 u}$ RESONANCE AT $1.15 \mathrm{eV}$ AND OVERTONE EXCITATION}

While recording the experimental data for this publication, we necessarily obtained information on two phenomena currently not accessible to the present DMR method, and, to our knowledge, no other theory. They are (a) the boomerang structure of the ${ }^{2} \mathrm{e}_{2 \mathrm{u}}$ lowest $\pi^{*}$ resonance and (b) the excitation of overtone and combination vibrational states. We shall report this experimental information in this section because even a qualitative discussion of these data yields conclusions relevant to this paper and provides experimental information to guide future theoretical work.

The qualitative interpretation of the experimental data will be based on the following rules:

(i) Infrared (IR) active modes may be excited directly by the dipole mechanism. The cross sections due to this mechanism peak at threshold and then steeply decrease within a few tenths of an $\mathrm{eV}$. The mechanism may be described theoretically by the Born approximation. This threshold peak is absent for IR inactive vibrations, that is, for IR inactive modes and for transition to overtone and combination vibrational states.

(ii) Cross sections for infrared inactive modes, including overtone and combination vibrations, are useful for identifying resonances because the background of direct excitation is absent. 
(iii) Shorter-lived resonances, i.e., resonances with a large electronic width $\Gamma$, excite overtones relatively less than they excite fundamentals because excitation of overtones requires large nuclear relaxation, not possible during the short interaction time of short-lived resonances. (Note that the experimentally observed widths of EDS peaks are combinations of the electronic widths $\Gamma$ and the FranckCondon widths and thus do not provide direct information about $Г$.) The relative heights of resonant peaks in the ED spectrum of an overtone, as compared to the spectra of the corresponding fundamental, thus provide qualitative information on the relative electronic widths $\Gamma$ of the resonances.

(iv) Vibrational modes along which the potential surface of the temporary anion is strongly repulsive at the point of attachment are strongly excited.

\section{A. Boomerang structure within the first $\pi^{*}$ resonance and the threshold region}

The first step is to identify which are the prominent energy losses for which the cross sections should be recorded. For this purpose, we recorded an energy-loss spectrum at an incident electron energy corresponding to the vibrational origin of the $\mathrm{e}_{2 u}$ resonance and show it in Fig. 9. It is analogous to the $\mathrm{C}_{6} \mathrm{D}_{6}$ spectrum of Wong and Schulz ${ }^{2}$ except that they measured $\mathrm{C}_{6} \mathrm{D}_{6}$ at $70^{\circ}$. The peaks are identified by their apparent (i.e., taken from the spectrum, within $\pm 5 \mathrm{meV}$ ) energy loss in Fig. 9 because several overlapping modes are sometimes responsible for one peak. [The peak labeled " $2 \times 123$ " contains the first overtones of all modes whose fundamentals are contained in the peak labeled " 123 ," " $123+51$ " contains the combination vibrations of any of the modes whose fundamentals are

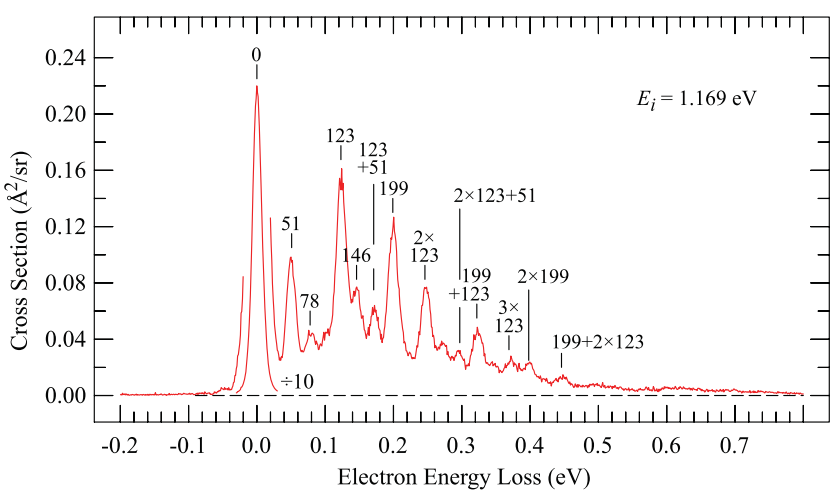

FIG. 9. Electron energy loss spectrum for the incident electron energy of $1.169 \mathrm{eV}$ recorded at $90^{\circ}$. The numbers above the spectrum indicate the observed energy losses of the peaks, in meV.

contained in the peak labeled " 123 " combined with one quantum “51" $\left(v_{20}\right)$, etc.]

In the second step, cross sections for selected energy losses were recorded as a function of the incident electron energy, and representative results are shown in Fig. 10. made:

The following comments based on the spectra in Fig. 10 can be

- The electronic width of the $\mathrm{e}_{2 \mathrm{u}}$ resonance, given by the width of the lowest boomerang peak, is $75 \pm 10 \mathrm{meV}$.

- The width of the entire progression of the boomerang peak (the Franck-Condon width of the peak) depends on which mode is excited. It is widest for exciting $v_{16}$ and narrowest for exciting the $\Delta E=123 \mathrm{meV}$ group of modes. We
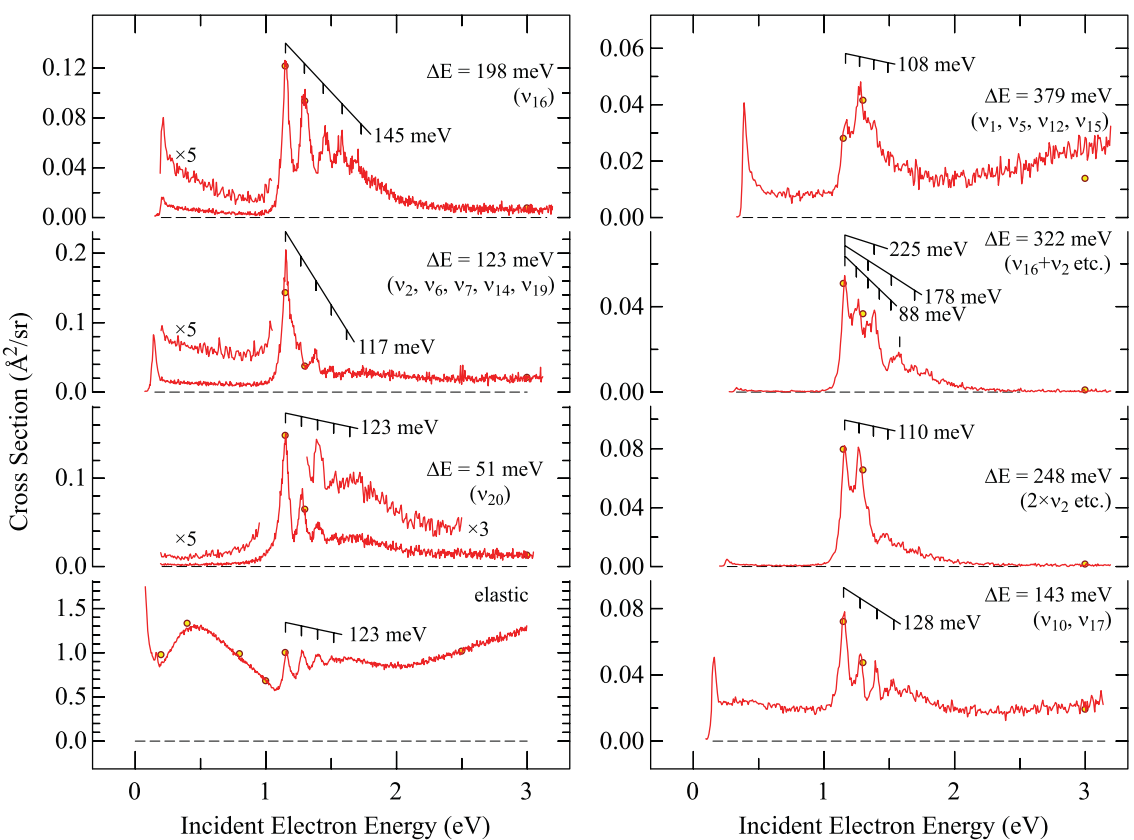

FIG. 10. Vibrational excitation cross sections recorded at $135^{\circ}$ for the fixed energy losses corresponding to the fundamental frequencies of the normal modes and for the overtone and combination bands as indicated. Boomerang progressions are indicated by grids above the spectra, with indication of the observed spacings. 
interpret these widths as the Franck-Condon widths of the attachment peak, that is, as reflecting the slope of (the real part of) the resonant potential hypersurface at the point of attachment-a steep potential hypersurface at the point of attachment leads to a wide peak.

- The spacings of the boomerang peaks also depend on the mode excited. They are $123 \mathrm{meV}$ for the elastic cross section and the $v_{20}$ excitation but attain other values (though all in the same ballpark) for exciting the other energy losses.

- The bands for exciting fundamental frequencies are dominated by single progressions. The bands for exciting the overtone $(\Delta E=248 \mathrm{meV})$ and combination $(\Delta E=322 \mathrm{meV})$ vibrational states have more complex shapes, defying simple assignment to progressions. Thus starts with two peaks 110 $\mathrm{meV}$ apart, of equal height, followed by an abrupt drop of cross section, not fitting any expected Franck-Condon profile. The band with $\Delta E=322 \mathrm{meV}$ is also complex. Two progressions, 88 and $178 \mathrm{meV}$, can be discerned. A peak is found at $225 \mathrm{meV}$ and another is found at $410 \mathrm{meV}$, both without discernible progressions, the latter being higher than any possible vibration.

- Threshold peaks are observed which are largely compatible with an assignment to Born-type direct dipole excitation. The vibrations $v_{6}, v_{14}$, and $v_{19}$ in the $\Delta E=123 \mathrm{meV}$ group are IR active, explaining the threshold peak in the $\Delta E=123 \mathrm{meV}$ cross section. $v_{10}$ is IR active, albeit weak, in the $\Delta E=143 \mathrm{meV}$ group. $v_{5}$ is IR active (very weak) and $v_{12}$ is IR active (strong), in the $\Delta E=379 \mathrm{meV}$ group, explaining the large threshold peak (note that $v_{12}$ is split to two peaks at 376 and $382 \mathrm{meV}$ due to a Fermi resonance with $v_{13}$ $+v_{16}$, but both components contribute to the $\Delta E=379 \mathrm{meV}$ group with our resolution). $v_{16}$ is IR inactive, and there is only a small threshold peak, which could be due to a small transition dipole due to anharmonicity. $\left(v_{20}\right.$ is also strongly IR active, but our spectrum in Fig. 10 does not extend to sufficiently low energy to show the threshold peak.) The overtone and combination vibrations yield only very small threshold peaks, which also could be due to a small transition dipole due to anharmonicity.

- The cross sections in the range from just above the Born peak to just below the $e_{2 u}$ resonance (about $0.5-1.0 \mathrm{eV}$ ) are peculiar. They are much smaller than those caused by the (very strong) $\mathrm{e}_{2 \mathrm{u}}$ resonance but still quite substantial (they are high above the instrumental background). These cross sections cannot be assigned to the Born mechanism-they descend far too slowly (in the case of $\nu_{16}$ ) or are even constant (in the case of the peak with $\Delta E=123 \mathrm{meV}$, with several unresolved vibrations). The excitation is selectiveit is weak in the $v_{20}$ cross section and much stronger in the $\mathrm{C}-\mathrm{H}$ stretch excitation. The cross sections in this energy range are nearly zero for the overtone and combination vibration excitations, $\Delta E=248$ and $322 \mathrm{meV}$. The mechanism of this excitation is uncertain. It could be due to the low-energy tail of the $\pi^{*} e_{2 u}$ resonance.

A final interesting question is what vibrational modes are excited following an attachment of an electron not to the $v=0$ level of the resonance, like in Fig. 9, but to a higher level. Figure 11

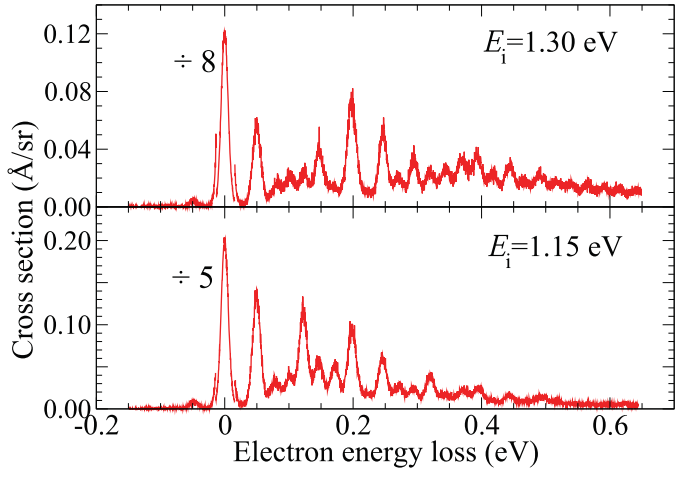

FIG. 11. Electron energy loss spectra for the incident electron energies of 1.15 and $1.3 \mathrm{eV}$ recorded at the scattering angle of $135^{\circ}$

addresses this question by comparing energy-loss spectra obtained at $E_{i}=1.15 \mathrm{eV}$ and $1.3 \mathrm{eV}$ (this time at $135^{\circ}$ ). The experimental answer is that a much higher density of overtone and combination vibrations is excited, in particular, at higher energy losses. It is important to note that this gradual loss of selectivity does not interfere with the postulation of the selectivity rules by Wong and Schulz ${ }^{2}$ - those rules were expressly postulated to apply only for electron attachment to the $v=0$ level of the resonance, with a totally symmetrical vibrational wave function in the transient anion. Figure 11 shows that the question of "selection rules" is closely linked, at least for resonances with the boomerang structure, to the incident electron energy and the symmetry of the intermediate (boomerang) wave function of the nuclei.

The qualitative picture to rationalize these observations is based on the calculation of Chourou and Orel, ${ }^{38}$ who calculated the motion of a nuclear wavepacket, in three dimensions, on an $a b$ initio complex potential hypersurface, to obtain a quantitative dissociative electron attachment cross section in acetylene. In our case, the nuclear wavepacket does not reach the dissociation point but decays by autodetachment into a vibrationally excited state of the target molecule. In the benzene case, the situation is fundamentally similar but more complex because of the many dimensions involved and because of the Jahn-Teller effect. The Jahn-Teller active modes are along the $e_{2 g}$ coordinate which should let it descent to the $D_{2 h}$ point group, but vibronic coupling with $\sigma^{*}$ states further changes the shape of the resonant potential surface, leading to a $C_{2 v}$ (Ref. 39) or even $C_{2}$ (Ref. 40) ground state of the benzene anion. The process of nuclear wavepacket relaxation appears to be accompanied by intramolecular vibrational redistribution (IVR) whereby the initially vibrationally selective electron attachment at higher energies within the resonance is converted to vibrational states with many overtones and combinations excited.

\section{B. Overtone excitation at higher energies}

Wide energy range spectra of the excitation of the $\Delta E=123 \mathrm{meV}$ and the $\Delta E=380 \mathrm{meV}$ groups of fundamental vibrations and of their first overtones at $\Delta E=245 \mathrm{meV}$ and $\Delta E=745 \mathrm{meV}$ are shown in Fig. 12. The value of $745 \mathrm{meV}$ is less than twice the fundamental frequency of $380 \mathrm{meV}$ because of anharmonicity and was 


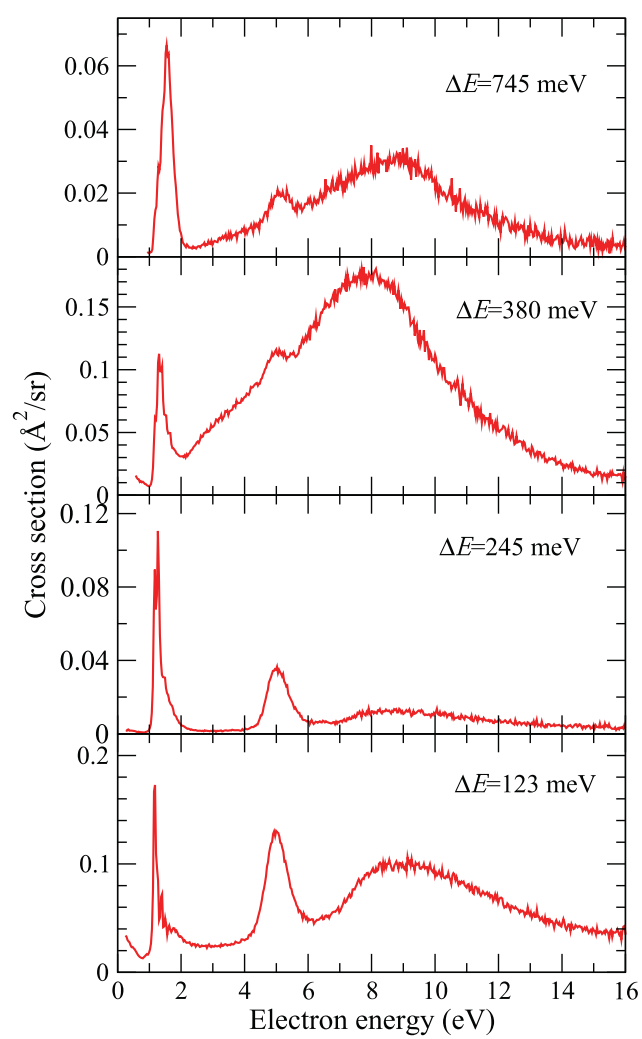

FIG. 12. Energy dependence spectra recorded at the scattering angle $90^{\circ}$ for fixed energy losses corresponding to frequencies of normal modes at 123 and $380 \mathrm{meV}$ and their first overtones.

determined from an energy-loss spectrum recorded at a constant residual energy of $8 \mathrm{eV}$, where a progression of peaks at 380, 745, and $1095 \mathrm{meV}$ appears. We do not know to which of the overlapping modes $v_{1}, v_{5}, v_{12}$, and $v_{15}$ does the energy loss peak of $745 \mathrm{meV}$ belong. made:

The following comments based on the spectra in Fig. 12 can be

- The height of the peak at $4.8 \mathrm{eV}$, relative to the $\mathrm{e}_{2 \mathrm{u}}$ peak at $1.15 \mathrm{eV}$, decreases when going from the $\Delta E=123 \mathrm{meV}$ spectrum to the $\Delta E=245 \mathrm{meV}$ spectrum. This is in line with a shorter lifetime of the $b_{2 g}$ resonance as compared to the $\mathrm{e}_{2 \mathrm{u}}$ resonance. This is also in line with the absence of the boomerang structure in this peak, although note that the absence of the boomerang structure can also be a consequence of a repulsive potential hypersurface.

- Similarly, the height of the very broad peak at 8-9 eV, relative to both the $\mathrm{e}_{2 \mathrm{u}}$ peak at $1.15 \mathrm{eV}$ and the $\mathrm{b}_{2 \mathrm{~g}}$ peak at $4.8 \mathrm{eV}$, decreases when going from fundamentals to overtones, indicating very short lifetime (large $\Gamma$ ) of the $\sigma^{*}$ resonances. Note that the width of the peak in the spectrum cannot be taken as the electronic width $\Gamma$ because it is likely to be caused in part by the Franck-Condon width associated with strongly repulsive hypersurfaces.
Interestingly, there is a nonzero cross section in the $2-4 \mathrm{eV}$ energy range, both in the $\Delta E=123 \mathrm{meV}$ and $\Delta E=380 \mathrm{meV}$ spectra, although this range is nominally between the $\mathrm{e}_{2 \mathrm{u}}$ and the $\mathrm{b}_{2 \mathrm{~g}} \pi^{*}$ resonances. This signal disappears for the overtone $\Delta E=245 \mathrm{meV}$ but persists in the $\Delta E=745 \mathrm{meV}$ overtone excitation, proving its resonant origin. Note that this weak cross section is reproduced by our calculation, as shown for the mode $v_{7}$ in Fig. 3 . We assign it to a very broad $\mathrm{e}_{1 \mathrm{u}}$ resonance.

\section{SUMMARY AND CONCLUSIONS}

In this paper, we tested the capacity of the Discrete Momentum Representation (DMR) method to describe resonances in electronmolecule scattering and, in particular, whether it correctly describes the coupling between the electron and nuclear motion, in the sense whether it correctly describes which normal modes are excited by which resonances and with what cross sections. In a broader sense, this coupling is essential for the description of electron-driven chemistry in the gas phase.

Limited resolution of the experiment does not permit measurements of cross sections for all vibrational modes individually, but experimental spectra were compared with theoretical profiles showing appropriate sums of cross sections for overlapping vibrations. Both the shapes and the heights of the profiles were compared since both the theory and the experiment yielded absolute values of the cross sections.

The DMR theory succeeds best for electron energies corresponding to resonances without narrow boomerang structure. Thus, we obtain a nearly quantitative agreement between the calculated and measured spectral profiles of energy-losses at the incident electron energy of $3 \mathrm{eV}$, both in terms of shape and of absolute magnitude. Satisfactory agreement was obtained also at 4.8 and $8 \mathrm{eV}$. Peaks in plots of cross sections are plotted as a function of energy reveal resonances. The DMR theory correctly reproduced the energies of the $\pi^{*}$ resonances. The DMR theory does not treat nuclear motion of the temporary anion and thus does not reproduce the experimentally observed boomerang vibrational structure of the first $\pi^{*}$ resonance and calculates it as a single peak. Although the theory calculated the resonance at the correct energy and with a qualitatively correct area under the peak in the elastic scattering, our calculations are not compatible with the strong excitation of $v_{2}$ reported by Wong and Schulz, ${ }^{2}$ and thus, the mechanism of $v_{2}$ excitation by the first $\pi \mathrm{e}_{2 \mathrm{u}}$ resonance in benzene remains an unresolved problem. The DMR theory qualitatively correctly reproduced the energies of the $\sigma^{*}$ resonances-the broad peaks in the $5-15 \mathrm{eV}$ region. The calculated resonances appear to be somewhat narrower than the experiment and appear as separate peaks, whereas the experiment shows only one very broad peak. The symmetry of the lower $\sigma^{*}$ resonance at $7.5 \mathrm{eV}$ was determined as $\mathrm{e}_{1 \mathrm{u}}$ in agreement with the work of Gianturco and Lucchese ${ }^{12}$ who computed it at $10.07 \mathrm{eV}$. The symmetry of the other $\sigma^{*}$ resonance at $10.5 \mathrm{eV}$ was identified as $\mathrm{e}_{2 \mathrm{~g}}$. We notice that resonances can be discerned much more clearly in plots of vibrational excitation cross sections than in plots of elastic cross sections. This means that the DMR theory, providing vibrational excitation cross sections, is more powerful in identifying shape resonances than theories calculating elastic cross sections alone. Of key importance is the applicability of the DMR theory even to large polyatomic molecules, including molecules of practical importance. As 
an example, work is in progress on the molecule $\mathrm{Fe}(\mathrm{CO})_{5}$, relevant for nanolithography.

Our experimental spectra in Figs. 7 and 9 are fully compatible (isotopic shifts of vibrational frequencies taken into account) with the $\mathrm{C}_{6} \mathrm{D}_{6}$ energy loss spectra at 1.15 and $4.8 \mathrm{eV}$ of Wong and Schulz. ${ }^{2}$ Our work shows, however, that the selection rules which they propose and which were later treated in more depth ${ }^{3}$ are essentially correct as the first approximation but that the situation is more complex at a closer look, in particular, that more modes are excited than predicted by the selection rules by Wong and Schulz. ${ }^{2}$ We pointed this out already in our earlier work on cyclopropane, ${ }^{1}$ where vibrational modes were excited requiring that an electron arriving in an $\mathrm{f}$ wave departs in a p-wave, thus extending the postulate of Wong and Schulz that the electron can depart either in the same partial wave in which it arrived or in an s-wave. Note that Wong and Schulz were cautious in their formulation of the selection rules in the sense that they apply only when the vibrational wave function of the transient anion is totally symmetric, that is, $v=0$, reached with an incident electron energy of $1.15 \mathrm{eV}$. Their selection rules were thus expressly not intended to cover higher incident energies, like in the top panel of Fig. 11. Note also that our results at $4.8 \mathrm{eV}$ show a less dramatic selection of modes than assumed by Wong and Schulz. Our calculations suggest that the $4.8 \mathrm{eV}$ resonance does not excite just a single $\mathrm{CH}$ stretching mode $\left(\mathrm{a}_{1 \mathrm{~g}} v_{1}\right)$ and also not just a single $\mathrm{CH}$ bend $\left(\mathrm{b}_{2 \mathrm{~g}} v_{7}\right)$.

Our work shows that the selection rules of Wong and Schulz, while valuable for qualitative assessment, oversimplify the excitation process and cannot replace a quantitative calculation like the present one.

We complemented the above work with experimental results on boomerang structure in the first $\pi^{*}$ resonance and on excitation of overtones. The cross sections for overtone excitation point out that the intensity of the third $\pi^{*}$ resonance at $4.8 \mathrm{eV}$, relative to the first and second resonance (degenerate at $1.15 \mathrm{eV}$ ), decreases in the overtone spectrum, reflecting its larger width. Similarly, the very broad $\sigma^{*}$ peak in the $8-10 \mathrm{eV}$ range decreases faster than all $\pi^{*}$ resonances, indicating an even larger width $\Gamma$.

The boomerang structure within the $1.15 \mathrm{eV}$ resonance is dominated by spacings of around $123 \mathrm{meV}$ which vary as a function of which vibrational mode is being excited. The width and shape of the boomerang profile, that is, the number and relative intensity of the boomerang overtones, vary dramatically in dependence for which the final mode is the cross section recorded. Increasing the incident energy within the boomerang profile, for example, from $1.15 \mathrm{eV}$ to $1.3 \mathrm{eV}$, dramatically increases the number of overtones and density of states excited after the departure of the electron. These results point out complex nuclear dynamics on the resonant potential surface, which is further complicated by Jahn-Teller distortion, and indicate extensive intramolecular vibrational redistribution (IVR) when vibrational energy is available in the transient benzene anion.

\section{ACKNOWLEDGMENTS}

R.Č. and P.Č. wish to thank the Grant Agency of the Czech Republic (Grant No. 18-02098S) for support of this work. M.A. acknowledges support from the Swiss National Science Foundation (Project No. 200020-144367/1).

\section{REFERENCES}

${ }^{1}$ R. Čurík, P. Čársky, and M. Allan, J. Chem. Phys. 142, 144312 (2015).

${ }^{2}$ S. F. Wong and G. J. Schulz, Phys. Rev. Lett. 35, 1429 (1975).

${ }^{3}$ G. A. Gallup, J. Chem. Phys. 99, 827 (1993).

${ }^{4}$ D. Prajapati, H. Yadav, P. C. Vinodkumar, C. Limbachiya, A. Dora, and M. Vinodkumar, Eur. Phys. J. D 72, 210 (2018).

${ }^{5}$ L. Sanche and G. J. Schulz, J. Chem. Phys. 58, 479 (1973).

${ }^{6}$ I. Nenner and G. J. Schulz, J. Chem. Phys. 62, 1747 (1975).

${ }^{7}$ P. D. Burrow, J. A. Michejda, and K. D. Jordan, J. Chem. Phys. 86, 9 (1987).

${ }^{8}$ P. Moźejko, G. Kasperski, C. Szmytkowski, G. P. Karwasz, R. S. Brusa, and A. Zecca, Chem. Phys. Lett. 257, 309 (1996).

${ }^{9}$ C. Makochekanwa, O. Sueoka, and M. Kimura, Phys. Rev. A 68 , 032707 (2003).

${ }^{10}$ M. Allan, J. Electron Spectrosc. Relat. Phenom. 48, 219 (1989).

${ }^{11}$ H. Cho, R. J. Gulley, K. Sunohara, M. Kitajima, L. J. Uhlmann, H. Tanaka, and S. J. Buckman, J. Phys. B: At., Mol. Opt. Phys. 34, 1019 (2001).

${ }^{12}$ F. A. Gianturco and R. R. Lucchese, J. Chem. Phys. 108, 6144 (1998).

${ }^{13}$ M. H. F. Bettega, C. Winstead, and V. McKoy, J. Chem. Phys. 112, 8806 (2000).

${ }^{14}$ R. Azria and G. J. Schulz, J. Chem. Phys. 62, 573 (1975).

${ }^{15}$ H. Kato, M. Hoshino, H. Kawahara, C. Makochekanwa, S. J. Buckman, M. J. Brunger, H. Cho, M. Kimura, D. Kato, H. A. Sakaue, I. Murakami, T. Kato, and H. Tanaka, National Institute for Fusion Science, NIFS-DATA-105, 2009.

${ }^{16}$ T. N. Rescigno, W. A. Isaacs, A. E. Orel, H.-D. Meyer, and C. W. McCurdy, Phys. Rev. A 65, 032716 (2002).

${ }^{17}$ A. Schramm, I. I. Fabrikant, J. M. Weber, E. Leber, M.-W. Ruf, and H. Hotop, J. Phys. B: At., Mol. Opt. Phys. 32, 2153 (1999).

${ }^{18}$ T. Nishimura and Y. Itikawa, J. Phys. B: At., Mol. Opt. Phys. 28, 1995 (1995).

${ }^{19}$ A. Jain and D. G. Thompson, J. Phys. B 17, 443 (1983).

${ }^{20}$ S. C. Althorpe, F. A. Gianturco, and N. Sanna, J. Phys. B: At., Mol. Opt. Phys. 28, 4165 (1995).

${ }^{21}$ R. Čurík and F. A. Gianturco, J. Phys. B: At., Mol. Opt. Phys. 35, 1235 (2002).

${ }^{22}$ M. Allan, J. Phys. B: At., Mol. Opt. Phys. 40, 3531 (2007).

${ }^{23}$ M. Allan, Phys. Rev. A 81, 042706 (2010).

${ }^{24}$ R. Čurík, I. Paidarová, M. Allan, and P. Čársky, J. Phys. Chem, A 118, 9734 (2014).

${ }^{25}$ R. K. Nesbet, Phys. Rev. A 20, 58 (1979).

${ }^{26} \mathrm{P}$. Čársky and R. Čurík, in Low-Energy Electron Scattering by Molecules, Biomolecules and Surfaces, edited by P. Čársky and R. Čurík (CRC Press; Taylor\&Francis Group, 2012), p. 263.

${ }^{27}$ P. Čársky and R. Čurík, Theor. Chem. Acc. 134, 102 (2015).

${ }^{28}$ R. Čurík and M. Šulc, J. Phys. B: At., Mol. Opt. Phys. 43, 175205 (2010).

${ }^{29}$ J. Sadlej, Collect. Czech. Chem. Commun. 53, 1955 (1988).

${ }^{30}$ See http://daltonprogram.org for Dalton, a molecular electronic structure program, Release 2.0, 2005

${ }^{31}$ T. Shimanuchi, Tables of Molecular Vibrational Frequencies Consolidated (National Bureau of Standards, 1972), Vol. 1, p. 1.

${ }^{32}$ D. M. Chase, Phys. Rev. 104, 838 (1956).

${ }^{33}$ A. Herzenberg, J. Phys. B: At., Mol. Opt. Phys. 1, 548 (1968).

${ }^{34}$ N. Chandra and A. Temkin, Phys. Rev. A 13, 188 (1976).

${ }^{35}$ A. J. F. Siegert, Phys. Rev. 56, 750 (1939).

${ }^{36}$ O. I. Tolstikhin, V. N. Ostrovsky, and H. Nakamura, Phys. Rev. A 58, 2077 (1998).

${ }^{37}$ I. C. Walker, A. Stamatovic, and S. F. Wong, J. Chem. Phys. 69, 5532 (1978).

${ }^{38}$ S. T. Chourou and A. E. Orel, Phys. Rev. A 77, 042709 (2008).

${ }^{39}$ L. Andjelkovic, M. Peric, M. Zlatar, S. Grubišić, and M. Gruden-Pavlović, Tertrahedron Lett. 53, 794 (2012).

${ }^{40}$ A. P. Bazante, E. R. Davidson, and R. J. Bartlett, J. Chem. Phys. 142, 204304 (2015). 